\title{
FULLY AUTOMATED POINT-BASED ROBOTIC NEUROSURGICAL PATIENT REGISTRATION PROCEDURE
}

\author{
Suligoj, F.; Jerbic, B.; Svaco, M.\# \& Sekoranja, B. \\ Department of Robotics and Production System Automation, Faculty of Mechanical Engineering and \\ Naval Architecture, University of Zagreb, Zagreb, Croatia \\ E-Mail: filip.suligoj@fsb.hr, bojan.jerbic@fsb.hr, marko.svaco@fsb.hr, bojan.sekoranja@fsb.hr \\ ( ${ }^{\#}$ Corresponding author)
}

\begin{abstract}
In this study, we have introduced a framework for an automatic patient registration procedure using freely distributed fiducial markers within a robot application in neurosurgery. The localization procedures in the image space and in the physical space are fully automated. We have developed a novel algorithm for finding the point pair correspondence between freely distributed fiducial markers in the image and in the physical space. The algorithm introduces a similarity matrix to maximize the possibility of successful point pairing and to remove the potential outlier points. The correspondence algorithm has been tested in 900,000 computer simulations and also on the real data from five laboratory phantom CT scans and twelve clinical patient CT scans, which were paired with 1415 readings captured with an optical tracking system. Testing of simulated point scenarios showed that the correspondence algorithm has a higher percentage of success when a larger number of fiducial markers and a lower number of outlier points were present. In the 24055 tests on the clinical data, there has been a $100 \%$ success rate.

(Received in March 2018, accepted in June 2018. This paper was with the authors 1 week for 1 revision.)
\end{abstract}

Key Words: Biomedical Imaging, Medical Robotics, Iterative Algorithms, Iterative Closest Point Algorithm, RONNA

\section{INTRODUCTION}

There is a growing trend for applying robotic systems in different aspects of medical practice because of numerous benefits that they provide. Robotic systems coupled with medical imaging techniques (computed tomography - CT, magnetic resonance imaging - MRI, etc.) can provide great accuracy of positioning the surgical instrument on planned targets, repeatability of position and motion, ability to hold the position over a period of time without tremor, and programmability of complex motions. As with any technology-driven change, newer versions aim to improve their primary features, the efficiency, and the ease of use. Modern medical robotic systems and image-guided surgical procedures possess little autonomy or very few automated functions [1]. A great number of operations are still performed manually, which can be attributed to the unstructured working environment in the operating room $(\mathrm{OR})$ and to demanding safety requirements in robotic surgery.

State-of-the-art robotic systems intended for neurosurgery and their patient registration methods differ in regard to the employed patient localization features, localization techniques, sensors, and registration procedures. Fiducial markers which can be used as reference points are physical objects used in the localization process. Medical image registration techniques are used for aligning the image space with the physical space by calculating their rigid body transformation [2,3]. As shown by Widmann et al. [4], registration based on paired points is still the gold standard in frameless stereotactic neurosurgery. Nevertheless, in a number of patient registration procedures with robotic systems, corresponding points between the image and the physical space, either fiducial points or anatomical landmarks, need to be manually matched. This is a time consuming but also an error-prone process because the operator has to point out each fiducial marker by hand and confirm the choice. In comparison to manual 
methods which depend on the operator and the technology, the automatic correspondence can be achieved in a few milliseconds (depending on the correspondence algorithm and the number of points). Cardinale et al. [5] evaluate the new Neuromate (Renishaw, Gloucestershire, UK) Neurolocate touch-free localization device and its clinical workflow. After an intraoperative 3D image has been obtained with the O-arm, the centres of the Neurolocate fiducial markers need to be selected semi-automatically in multi-planar reconstructions within the planning software. In [6], Benedictis et al. reported on the ROSA Brain neuronavigation robotic assistant (Medtech, Montpellier, France) which can be used with two types of registrations: fiducial marker registration using bone-attached fiducials and frameless surface-based registration (i.e. noncontact patient localization). The first procedure is based on the manual positioning of the robot tool centre point (mechanical pointer) within the implanted screws on the patient's skull. González-Martínez et al. [7] and Lefranc et al. [8] used the ROSA Brain neuronavigation robotic system with the noncontact localization method utilizing a custom-built laser for measuring distance. The main drawback of this approach is that the initial alignment between the image space and the physical space is done by the operator, through manual guidance of the robotic arm, pinpointing a series of anatomical landmarks previously localized by the operator in the image space. Kronreif et al. [9] demonstrated a miniature robotic assistant system, B-RobII, which is navigated by a certified neuronavigation device (VectorVision, BrainLAB AG, Germany) using a manual registration procedure based on paired points. Gerber et al. [10] presented a semiautomatic ball-in-cone positioning method for the localization of fiducial markers in the physical space by using a novel surgical robotic system with force feedback control. In [11], Meng et al. present an optically tracked robotic system that utilizes a pointer tool and a patient reference frame in order to determine the correspondence between fiducial points in the physical and the image space. The same method of robotic guidance was used in our previous research [12]. Lin et al. [13] developed a neurosurgical robotic drilling and navigation system which utilizes manual localization of fiducial points in the physical space.

In this study, we propose a complete solution for achieving highest level of automation in the robotic neurosurgical patient registration procedure. To avoid the manual localization of fiducial markers in patient images, we use the previously developed automatic localization algorithm which calculates the coordinates of each fiducial marker [14]. An optical tracking system (OTS) Polaris Spectra (NDI - Northern Digital Inc., Ontario, Canada) with a large field of view is used for the localization of fiducial markers attached to the patient and the robot in the OR. For accurate point-based registration, it is crucial to determine the exact matching order and positions of all the fiducial markers. A novel algorithm for the point pair correspondence is described and evaluated. The algorithm uses a known positional mean error and the standard deviations of the input data from the OTS and a CT scanner with the localization algorithm to validate successful point pairing and to remove potential outliers. The successful point pairing between the patient image localization algorithm output and the OTS measurements ensures that there is no need for medical personnel to intervene in any phase of the patient registration procedure.

The paper is structured as follows. In section 2 we present the robotic system used in this study, the advantages of using the freely distributed markers, and disadvantages of manual localization. In section 3 we present all the phases of the robot clinical procedure and demonstrate transformations between all the coordinate systems which enable automation of the entire patient registration procedure. A state of the art in registration and correspondence algorithms and a novel algorithm for finding the point pair correspondence is described and evaluated in section 4. Finally, in section 5 we discuss the algorithm's test results and the plans for future research. 


\section{RESEARCH MOTIVATION}

Our research group has developed a dual-arm robotic system for frameless stereotactic neurosurgery, RONNA G3. The main system components, registration procedure, and the overall operating procedure were first presented in [15] and the position planning strategies which search for the optimal placement of a neurosurgical robot in [16]. In the registration procedure, a rigid bone-implanted x-shaped frame, shown in Fig. 1 a, was used for localization in the patient images (patient CT scan) and the robot coordinate system in the physical space. The $\mathrm{x}$-shaped frame is designed to carry four fiducial markers positioned at a unique distance from each other. During the first series of human clinical trials carried out in cooperation with a team of neurosurgeons from the University Hospital Dubrava (Klinička bolnica Dubrava KBD), we noticed that the $\mathrm{x}$-shaped frame implantation procedure proved to be impractical due to the frame size. An alternative was to replace the $\mathrm{x}$-shaped frame with three or more individual self-drilling and self-tapping screws shown in Fig. $1 \mathrm{~b}$. Thus, retro-reflective spheres, i.e. fiducial markers, can be attached to them. In this study, individual fiducial markers that are implanted on the head of the patient will be called freely distributed fiducial markers. Advantages of using freely distributed fiducial markers are:

- less invasiveness because of the smaller insertion diameter used for individual markers,

- better flexibility in choosing a position of fiducial markers on the patient's head in relation to the planned surgery target,

- simpler pre-operative procedure for implanting self-drilling and self-tapping screws,

- smaller registration error when individual fiducial markers are placed at larger distances,

- ability to use more than four fiducial markers if higher precision and accuracy are required,

- shorter distance from the fiducial markers to the surgical target.

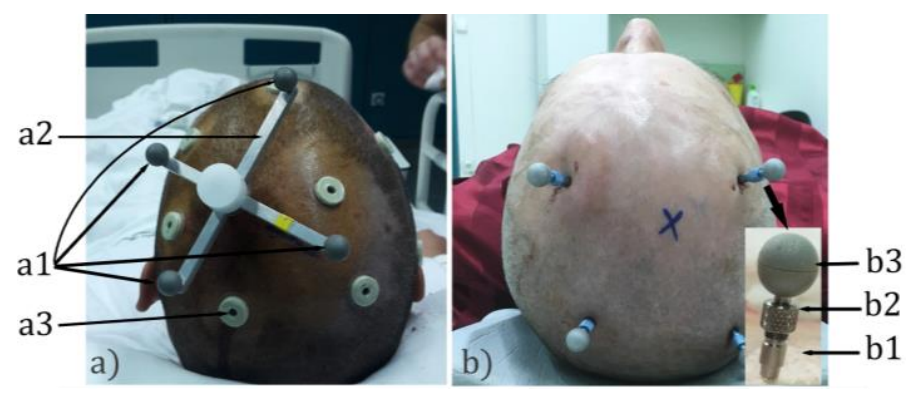

Figure 1: a) The bone-implanted $\mathrm{x}$-shaped frame $\mathbf{a 2}$ with four fiducial markers $\mathbf{a 1}$ and skin-attached fiducial markers a3; b) Freely distributed fiducial markers composed of: a self-drilling and self-tapping screw b1, a removable base $\mathbf{b 2}$, a retro-reflective sphere, i.e. a fiducial marker $\mathbf{b 3}$.

Freely distributed skin-attached fiducial markers shown in Fig. 1 a are also used in the OTS surgical navigation which is a standard medical method for the tracking and visualization of specially designed surgical tools relative to the real patient image. Surgical navigation by means of the OTS uses skin-attached fiducial markers that are freely distributed on the patient's skull. These fiducial markers are manually localized by the medical personnel in both the image space and the OR, and the correspondence between the associated point pairs is determined manually. The disadvantages of manual procedures are duration of localization, a possibility of a human error, insufficient accuracy, and staff training required to perform the localization procedure. The robot-guided interventions share a similar registration procedure with that of the OTS surgical navigation and hence share the same disadvantages if manual localization and registration are used. A fully automated patient registration procedure with a robot system using freely distributed fiducial markers requires automated localization procedures and an algorithm that can determine the corresponding point pairs between the image space and the physical space data sets. 


\section{RONNA G3 CLINICAL PROCEDURE FOR AUTOMATIC PATIENT REGISTRATION}

The RONNA G3 clinical procedure is composed of three phases: the preoperative phase, the preparation phase, and the operation phase, as shown in Fig. 2. In the following sub-sections, the clinical procedure is described with an emphasis on the automation of the patient registration procedure and the calculation of transformations between all coordinate systems.

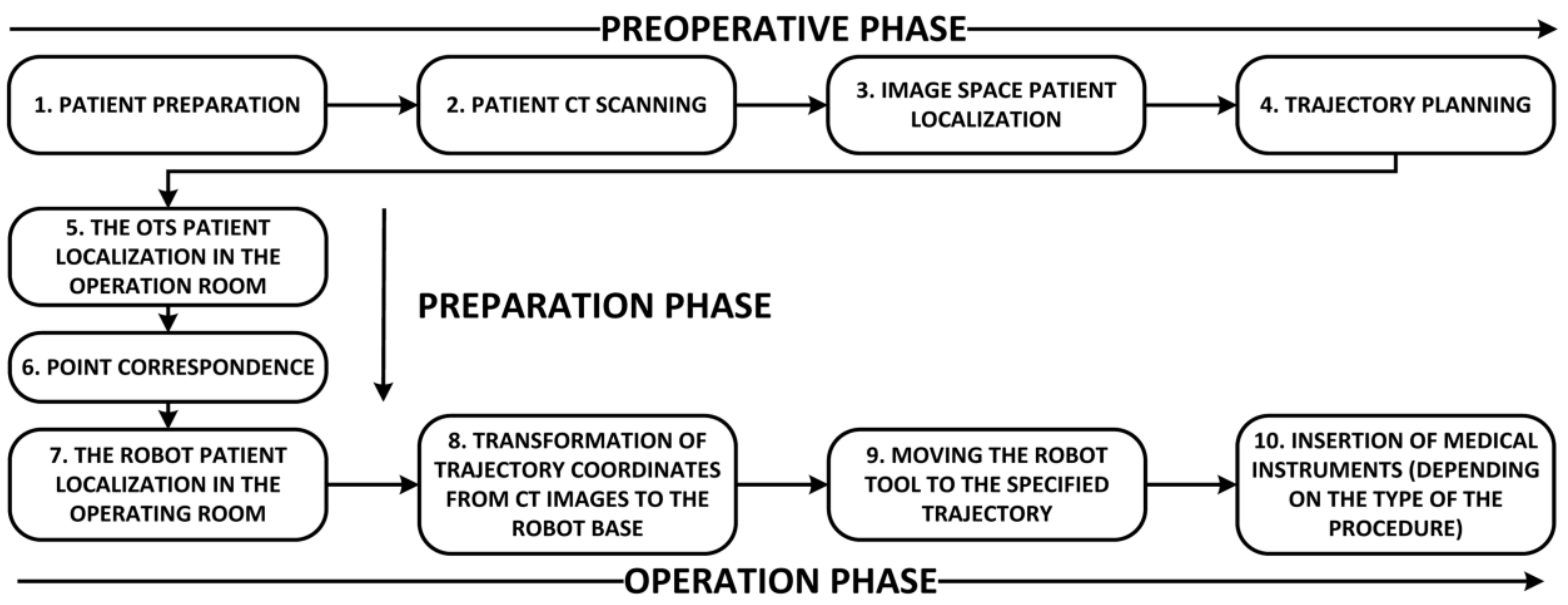

Figure 2: Technical workflow of the RONNA G3 clinical procedure.

A better general description of the RONNA G3 surgical procedure can be found in the paper by Švaco et al. [17] while a medical case in which the RONNA G3 system was used is presented in the paper by Dlaka et al. [18].

\subsection{Preoperative phase}

In the preoperative phase, the bone-attached screws are fixed to the patient's head and the patient is scanned with a CT scanner. During this phase, the positioning of individual fiducial markers in relation to one another is critical because only the right positioning guarantees successful point pairing in the later phases of the registration process. After scanning, the patient images are imported into the software for operation planning (RONNAplan) [17].

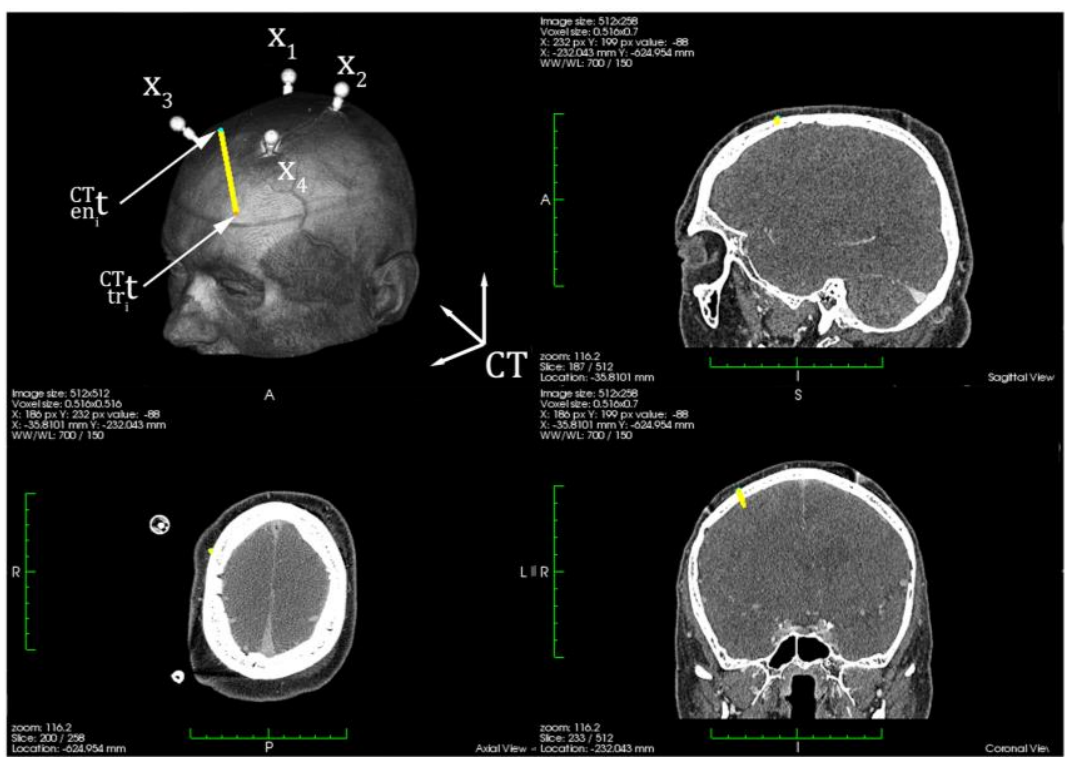

Figure 3: Trajectory definition in the RONNAplan planning software is shown with the freely distributed markers attached on the patient during clinical tests. 
Since the trajectory point coordinates are defined in the CT coordinate system, they need to be transformed to the coordinate system of the robot denoted as $\mathbf{R}$. The localization of fiducial markers in the patient images is the first step in the patient registration procedure. Automatic localization is achieved with our localization algorithm developed and described in [14]. The algorithm uses a unique approach combining machine vision algorithms, biomedical image filtration methods, and mathematical estimation methods to determine the centre of each individual fiducial marker. The output of the localization algorithm is a set of fiducial points $\left\{\boldsymbol{x}_{\boldsymbol{i}}\right\}$. RONNAplan enables visualization of the patient's anatomy and planning of $i=1, \ldots, a$ number of operation trajectories in the coordinate system of the CT scanner (denoted as CT). Each operation trajectory is composed of two points, an entry point $\mathbf{e n}_{\mathrm{i}}$ written as translation vector ${ }_{\mathrm{en}}^{\mathrm{CT}} \mathrm{t}$ defined on the surface of the patient's skull and the surgery target point $\mathbf{t r}_{\mathrm{i}}$ denoted as ${ }_{\mathrm{tr}_{\mathrm{i}}}^{\mathrm{CT}} \mathbf{t}$. An example of the planned trajectory is shown in Fig. 3.

\subsection{Preparation phase}

At the start of the surgery, in the preparation phase of the RONNA G3 procedure, the patient is brought to the OR and the robot is positioned near the patient. In the OR, an OTS, i.e. an infrared stereo camera system with a large operating volume, is used for the localization of fiducial markers placed on the patient and on the robot. At this phase, there are two separate point sets. The first set of $i=1, \ldots, n$ points $\left\{\boldsymbol{x}_{\boldsymbol{i}}\right\}$ are positions of the fiducial markers in the image space (CT). The second set of $j=1, \ldots, m$ points $\left\{\boldsymbol{y}_{j}\right\}$ are coordinates of the fiducial markers attached to the patient in the physical space captured by the OTS (coordinate system OTS). In this paper, every point $\mathbf{x}_{i}$ or $\mathbf{y}_{j}$ will also be denoted as translation vector ${ }_{x_{i}}^{C T} t_{y_{j}}$ or ${ }_{y_{j}}$ OTS $_{\text {, }}$ depending on the context. To solve the rigid point-based registration problem, the correspondence between $\left\{\boldsymbol{x}_{\boldsymbol{i}}\right\}$ and $\left\{\boldsymbol{y}_{j}\right\}$ must be established. Once at least three corresponding point pairs have been matched, the problem is reduced to calculating the $3 \times 3$ rotation matrix $\mathbf{P}$ and the $3 \times 1$ translation vector $\mathbf{t}$ that aligns the corresponding $l$ number ( $l$ can be smaller than $m$ and $n$ if the point set includes outliers) of points from $\left\{\boldsymbol{x}_{i}\right\}$ and $\left\{\boldsymbol{y}_{j}\right\}$ in a way which minimizes the root-mean-square $(R M S)$ distance between the points:

$$
d^{2}=\frac{1}{l} \sum_{i=1}^{l}\left|\mathbf{y}_{\mathrm{i}}-\left(\mathbf{P} \mathbf{x}_{\mathrm{i}}+\mathbf{t}\right)\right|^{2}
$$

Typically, because of the localization errors, the value of $d$ cannot be zero. An example of the method for the rigid point-based registration is the least squares fitting of two 3D point sets [6]. The final goal of the registration procedure is to find the transformation between the image space and the physical space for the OTS:

or for the robot:

$$
\underset{\mathrm{OTS}}{\mathrm{CT}} \mathbf{T}=\left[\begin{array}{cc}
\mathrm{OTS} \mathbf{P} & \mathrm{OTS}^{\mathrm{CT}} \mathbf{t} \\
0 & 1
\end{array}\right]
$$

$$
{ }_{\mathrm{R}}^{\mathrm{CT}} \mathbf{T}=\left[\begin{array}{cc}
{ }_{\mathrm{R}}^{\mathrm{CT}} \mathbf{P} & { }_{\mathrm{R}}^{\mathrm{CT}} \mathbf{t} \\
0 & 1
\end{array}\right]
$$

If the correspondence of points between the coordinate systems CT and OTS can be established, we can calculate the position of the patient in the robot coordinate system $\mathbf{R}$.

As can be seen in Fig. 4, the OTS which retrieves the coordinates of the fiducial markers is used to attain the position and the orientation of the dynamic reference frame $\mathbf{M}$ mounted on the robot tool $\left({ }_{\mathrm{M}}^{\mathrm{OTS}} \mathbf{T}\right)$ and the positions of the freely distributed fiducial markers $\left\{\boldsymbol{y}_{i}\right\}$ attached to the patient. $\mathbf{M}$ is retrieved in the OTS as ${ }_{\mathrm{M}}^{\mathrm{OTS}} \mathbf{T}$, i.e. the position and orientation of the predefined configuration of individual fiducials attached to the robot tool. 


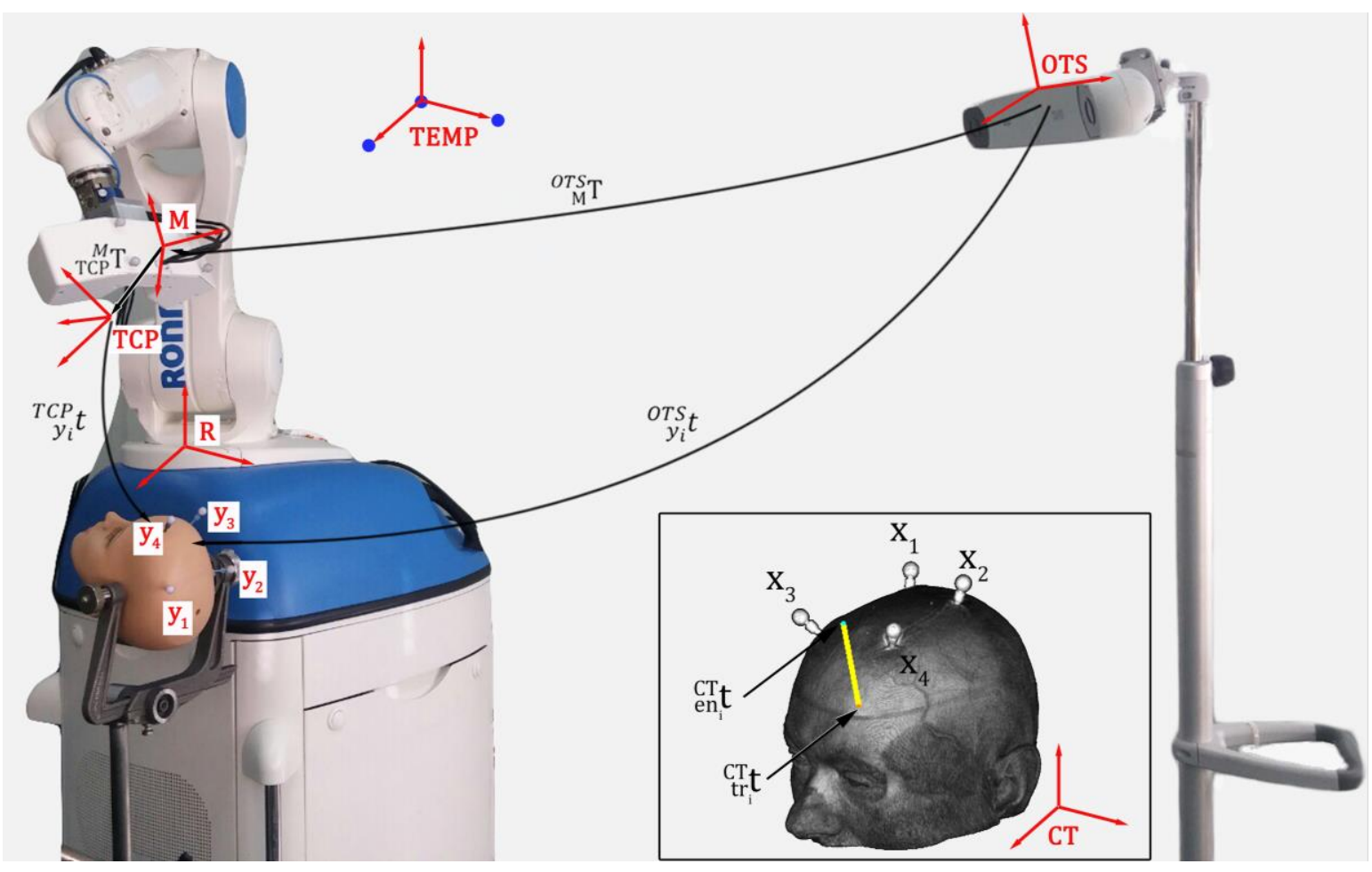

Figure 4: Coordinate systems and transformations used for achieving an automatic patient registration procedure.

Positions of the patient fiducial markers in the coordinate system of the OTS are the translation vectors which will be denoted as ${ }_{\mathrm{y}_{\mathrm{i}}} \mathrm{T}$. The connection between the position and orientation of the fiducial marker attached to the robot tool and the tool centre point (TCP) of the robot is defined by the transformation ${ }_{\mathrm{TCP}}^{\mathrm{M}} \mathbf{T}$. The transformation ${ }_{\mathrm{TCP}}^{\mathrm{OTS}} \mathbf{T}$ is calculated as:

$$
{ }_{\mathrm{TCP}}^{\mathrm{OTS}} \mathbf{T}={ }_{\mathrm{M}}^{\mathrm{OTS}} \mathbf{T} \cdot \underset{\mathrm{TCP}}{\mathrm{M}} \mathbf{T}
$$

The position and orientation of the pre-calibrated TCP in the robot base coordinate system $\left({ }_{T C P}^{R} \mathbf{T}\right)$ is aquired from the robot controller. The translational and rotational parts of the transformation ${ }_{\mathrm{TCP}}^{\mathrm{M}} \mathbf{T}$ are determined by means of tool calibration. The translation ${ }_{\mathrm{TCP}}^{\mathrm{M}} \mathbf{t}$ is first calculated by moving the TCP of the robot arm in several configurations around the same point in space and by using the method described in [19]. The orientation is calculated by moving the TCP of the robot in three points and by creating a new coordinate system TEMP in the space which is shared by the robot ${ }_{\text {TEMP }}^{\mathrm{R}} \mathbf{T}$ and the OTS ${ }_{\text {TEMP }}^{\mathrm{OTS}} \mathbf{T}$. For the calculation of rotation matrix $\underset{\operatorname{TPC}}{\mathrm{M}} \mathbf{P}$, the orientation of the marker captured by the OTS and the orientation of the TCP in the robot coordinate system are recorded at the same time.

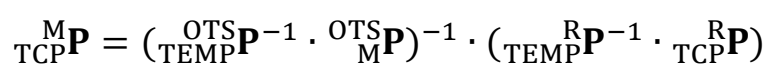

When ${ }_{\text {TCP }}^{\mathrm{M}} \mathbf{T}$ is known, we can calculate the value of ${ }_{\text {TCP }}^{\text {OTS }} \mathbf{T}$ using the Eq. (4) and determine the coordinates of the fiducial markers in the TCP coordinate system:

$$
{ }_{\mathrm{y}_{\mathrm{i}}}^{\mathrm{TCP}} \mathbf{t}={ }_{\mathrm{TCP}}^{\mathrm{OTS}} \mathbf{T}^{-1} \cdot \underset{\mathrm{y}_{\mathrm{i}}}{\mathrm{OT}} \mathbf{t}
$$

The coordinates of the fiducial markers in the $\mathbf{R}$ coordinate system are then calculated as:

$$
\underset{\mathrm{y}_{\mathrm{i}}}{\mathrm{R}} \mathbf{t}=\underset{\mathrm{TCP}}{\mathrm{R}} \mathbf{T} \cdot \underset{\mathrm{y}_{\mathrm{i}}}{\mathrm{TCP}_{\mathrm{t}}}
$$

on the assumption that the robot joint positions used for calculating $\underset{\operatorname{TCP}}{\mathrm{R}} \mathbf{T}$ are identical to the ones used when capturing ${ }_{\mathrm{M}}^{\mathrm{OTS}} \mathbf{T}$ and ${ }_{\mathrm{y}_{\mathrm{i}}}^{\mathrm{OTS}} \mathbf{t}$. Knowing the coordinates of the fiducial markers in 
the robot base coordinate system means that the patient position in relation to the robot is known. At this point, the preparation phase is completed.

\subsection{Operation phase}

In the operation phase, the fiducial marker localization by means of a robot is performed using the RONNAstereo device [17]. RONNAstereo consists of two infrared cameras with macro lenses (acA2000-50gmNIR, Basler, Ahrensburg, Germany). The two cameras are used to create the virtual TCP that corresponds with the TCP of the robot. The robot arm with the mounted RONNAstereo device is sent to every ${ }_{\mathrm{y}_{\mathrm{i}}}^{\mathrm{R}} \mathbf{t}$ and all the fiducial points are re-localized directly in the robot base coordinate system. Re-localization ensures better precision than the sole reliance on the OTS coordinates. After registration, the RONNAstereo is physically replaced with a surgical tool. The surgical tool can then be moved by the robot to any trajectory ${ }_{\mathrm{en}_{\mathrm{i}}}^{\mathrm{CT}} \mathbf{t} \rightarrow{ }_{\mathrm{tr}_{\mathrm{i}}}^{\mathrm{CT}_{\mathrm{t}}} \mathrm{t}$ planned by the surgeon in the preoperative phase.

\section{REGISTRATION AND CORRESPONDENCE BETWEEN POINT PAIRS}

Finding point pairs between two sets of points, $\left\{\boldsymbol{x}_{i}\right\}$ and $\left\{\boldsymbol{y}_{\boldsymbol{i}}\right\}$, which have the root-meansquare $(R M S)$ distance $d$ between the points equal to zero, would mean that both inputs have zero positioning errors and no outlier localized points. In actual situations, the positioning errors from input devices are a consequence of environmental signal noise, errors produced due to discretization of the input signal, and the resolution of the device itself. Registration algorithms used for the geometric alignment of 3D point data are a well-researched topic in the fields of robotics and computer vision. Bellekens et al. [20] give an overview of the stateof-the-art registration methods, such as Singular Value Decomposition (SVD), Principal Component Analysis (PCA), and Iterative Closest Point (ICP) algorithm [21] with its variants. These methods are mostly used for processing data collected from various 3D sensors. The SVD method uses the cross correlation matrix to calculate the optimal transformation (in the least squares sense) between two point clouds when the correspondence between point pairs is known. The PCA gives a rotation matrix when aligning the directions of the largest eigenvectors extracted from the covariance matrices of the two data sets. Since the PCA method is very sensitive to outlier points, it is generally used only as the first rough estimation of the initial transformation in other algorithms such as the ICP. The ICP algorithm guesses the point correspondences between the data sets based on the nearest neighbour approach and iteratively refines the transformation. After each iteration of the algorithm, the outliers are disregarded in order to improve the previous estimate of the transformation parameters.

For our problem, we needed a registration algorithm that can compute the transformation between two point sets containing up to ten points each, with noise from the input devices and with a possibility of outlier points in both sets. Sets of up to ten points are realistic in regard to the number of fiducial markers used in standard neurosurgical applications when neuronavigation systems are used. As a solution to our specific problem, we have developed a novel correspondence algorithm.

\subsection{The correspondence algorithm}

Since we register data sets in a medical environment, the solution to our particular correspondence problem has to ensure great safety. It is of paramount importance for the algorithm to get the correct correspondence between every point pair in the presence of noise and potential outlier points, and to determine with a high level of statistical confidence that the mathematical solution is unique. Furthermore, it is important that no actual fiducial 
markers are classified as outliers. From the perspective of computing speed, the extenuating circumstance is that the number of used fiducial markers is always relatively small as it ranges between three and eight. The algorithm is used for finding correspondence between a known number of points in $\left\{\boldsymbol{x}_{\boldsymbol{i}}\right\}$ with $i=1, \ldots, n$ points and in $\left\{\boldsymbol{y}_{j}\right\}$ with $j=1, \ldots, m$ points. It should be noted that the positions of the individual markers are registered with an error which is composed of the resolution of input devices and the localization error which can be written as in [22]:

$$
\begin{aligned}
& \mathbf{x}_{\mathrm{i}}=\widehat{\mathbf{x}}_{\mathrm{i}}+\mathbf{e}_{\mathbf{x i}} \\
& \mathbf{y}_{\mathrm{j}}=\widehat{\mathbf{y}}_{\mathrm{j}}+\mathbf{e}_{\mathbf{y j}},
\end{aligned}
$$

where $\widehat{\mathbf{x}}_{1}$ and $\widehat{\mathbf{y}}_{\mathrm{j}}$ are the true point coordinates and $\mathbf{x}_{\mathrm{i}}$ and $\mathbf{y}_{\mathrm{j}}$ are the coordinates from the patient images and the OTS, which contain their respective errors $\mathbf{e}_{\mathrm{xi}}$ and $\mathbf{e}_{\mathrm{yj}}$. The flow chart of the algorithm is shown in Fig. 5.

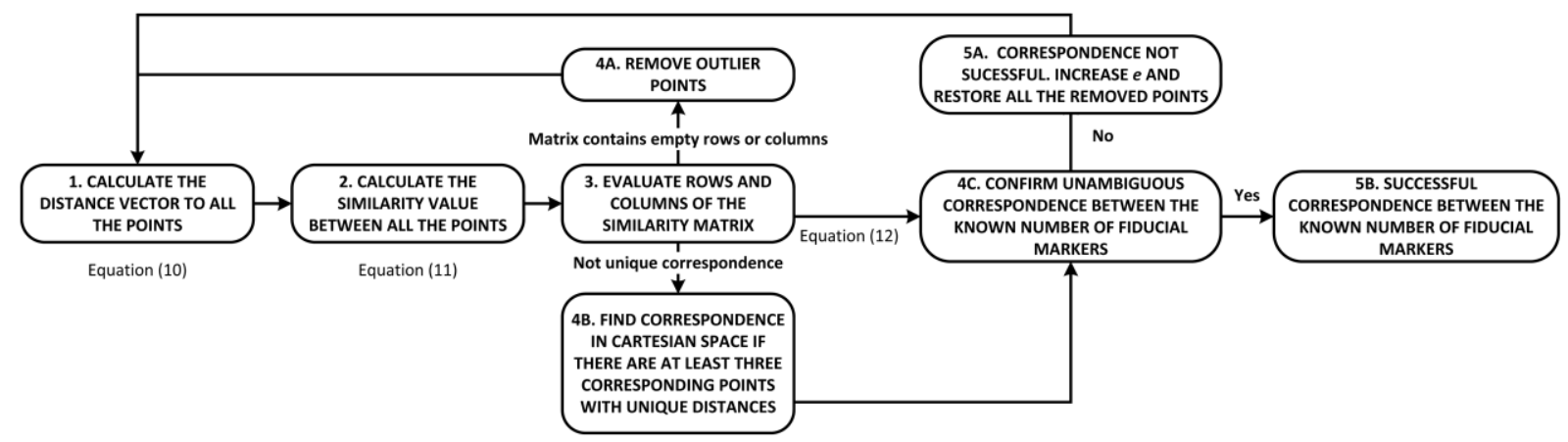

Figure 5: Correspondence algorithm flowchart.

In the first step of the algorithm, for every point in set $\left\{\boldsymbol{x}_{\boldsymbol{i}}\right\}$ and for every point in set $\left\{\boldsymbol{y}_{j}\right\}$, the Euclidean distance is calculated in relation to all the other points in the same point set. The Euclidean distance between any two points $\mathbf{x}_{1}$ and $\mathbf{x}_{2}$ is denoted as $d\left(\mathbf{x}_{1}, \mathbf{x}_{2}\right)$. As a result, every point in both data sets has a related vector containing distances to all the other points of the same data set:

$$
\mathbf{d}_{\mathbf{x}_{\mathbf{i}}}=\left[d\left(\mathbf{x}_{1}, \mathbf{x}_{\mathrm{i}}\right), \quad d\left(\mathbf{x}_{2}, \mathbf{x}_{\mathrm{i}}\right), \quad \ldots, \quad d\left(\mathbf{x}_{\mathrm{n}}, \boldsymbol{x}_{\mathrm{i}}\right)\right]
$$

In the second step, the similarity value $\operatorname{sim}_{i j}$ is calculated between all points $\mathbf{x}_{\mathrm{i}}$ in set $\left\{\boldsymbol{x}_{\boldsymbol{i}}\right\}$ and $\mathbf{y}_{\mathrm{j}}$ in set $\left\{\boldsymbol{y}_{j}\right\}$ :

$$
\operatorname{sim}_{i j}=\text { count_members }\left\{\left(\mathbf{d}_{\mathbf{x}_{\mathbf{i}}}-\mathbf{d}_{\mathbf{y}_{\mathbf{j}}}\right)<e\right\},
$$

where $e$ is the value of the largest difference allowed between any two values in $\mathbf{d}_{\mathbf{x}_{\mathbf{i}}}$ and $\mathbf{d}_{\mathbf{y}_{j}}$ that will be treated as a similarity point. The notation "count_members" in Eq. (11) is used for counting the number of shared distance instances between any two points in both data sets, and writing it into the similarity matrix. The minimum value that $\operatorname{sim}_{i j}$ can attain is zero and the maximum value is the number of points in the smaller data set minus one. The logic behind the similarity matrix is that for all the actual fiducial points in the one point set and all the distances between the points of the same set there should be the same number of fiducial points in the second set with the distances between those points smaller than the value of parameter $e$. There is one less distance instance in a vector $\mathbf{d}_{\mathbf{x}_{\mathbf{i}}}$ than the number of points in a set since $d\left(\mathbf{x}_{\mathrm{i}}, \mathbf{x}_{\mathrm{i}}\right)=0$. Since $e$ is the measure of acceptance between any two distances, it is in our interest to make it as small as possible for the algorithm to be able to differentiate between similar distances. On the other hand, if our input devices return data which contains measurement errors for a fiducial point position which is greater than $e$, then that fiducial point would be excluded and the correspondence cannot be established. For that reason, the 
interval of values for parameter $e$ is based on the measured error of the input devices. The calculated similarity values of $\operatorname{sim}_{i j}$ for every two points in the two data sets are used for creating a 2D similarity matrix:

$$
\left.\begin{array}{ccc}
\mathbf{x}_{1} & \ldots & \mathbf{x}_{\mathrm{n}} \\
\mathbf{y}_{1} \\
\vdots \\
\mathbf{y}_{\mathrm{m}} \\
\operatorname{sim}_{11} & \cdots & \operatorname{sim}_{1 n} \\
\vdots & \ddots & \vdots \\
\operatorname{sim}_{m 1} & \cdots & \operatorname{sim}_{m n}
\end{array}\right]
$$

In the third step shown in the flowchart, rows and columns of the similarity matrix are evaluated in comparison to the expected number of fiducials. If the number of rows and columns is equal to the expected number of fiducial markers and every point in $\left\{\boldsymbol{x}_{\boldsymbol{i}}\right\}$ has its unique pair in $\left\{\boldsymbol{y}_{j}\right\}$, the algorithm has been successful in finding correspondence between the paired points (steps 3-4C-5B). If one or more outlier points are found in any of the point sets, $\operatorname{sim}_{i j}$ values in rows and columns of those points will be lower than that of the fiducial points. Every row or column of the matrix that does not have at least one value that satisfies the condition is then removed (step 4A). This marks the end of one iteration of the algorithm. The algorithm iterates the procedure in steps $1-4 \mathrm{~A}$ with a reduced number of points until it removes all the outliers or until the solution is confirmed as not being mathematically unique. Step 4B is initiated if the number of rows and columns in the similarity matrix is equal to the expected number of fiducial markers, but all the points do not have only one possible solution. In that case, the matrix is evaluated to find at least three corresponding points that are unique so that all the other point coordinates can be transformed to the coordinate systems of corresponding points (one for $\left\{\boldsymbol{x}_{\boldsymbol{i}}\right\}$ and one for $\left\{\boldsymbol{y}_{i}\right\}$ ) and compared in the Cartesian space. All the transformed points are checked in order to find their pairs based on their coordinates; the Euclidean distance between them should be smaller than $e$. The number of unique point pairs is then verified in step $4 \mathrm{C}$. If the unique correspondence cannot be found due to great errors or too many outlier points, the algorithm increases the allowed error $e$ (step 5A) and iterates through steps 1-3.

When freely distributed fiducial markers are used, there is a possibility of placing the markers at a similar distance from one to the other or symmetrically, which prevents achieving a unique mathematical solution for the correspondence between paired points of fiducials. Simple examples of such a setup are: three markers are used and placed in the positions to form a perfect equilateral triangle and four markers forming a square. In the case of mathematical insecurity for point-pairing, the registration cannot be performed automatically based only on the coordinates of the points and additional information is needed to resolve the ambiguous situation. In those situations, our algorithm relies on the manual intervention of the human operator who uses a specially designed pointer tool to point all fiducial markers.

\subsection{Correspondence algorithm testing in a simulated point environment}

The testing of the correspondence algorithm requires the input data to be modelled with realistic configurations of the fiducial markers and the measured distribution of fiducial localization errors. The fiducial localization error $(F L E)$ is defined as a distance between the true and the measured position of fiducial markers, as shown in Eqs. (8) and (9). In general, the FLE in the patient images is a result of noise produced by imaging artifacts, the resolution of the reconstructed images produced by the CT or the MR scanner, and the accuracy of the localization method [23, 24]. In our study, the FLE is generated by a CT scanner, an OTS, and by their localization methods. The zone of the potential $F L E$ from both input devices is shown in Fig. 6. It should be noted that the zone of localization error can be a circle/sphere (2D/3D) if the error distribution is isotropic or an ellipse/ellipsoid (2D/3D) if the error distribution is anisotropic. 


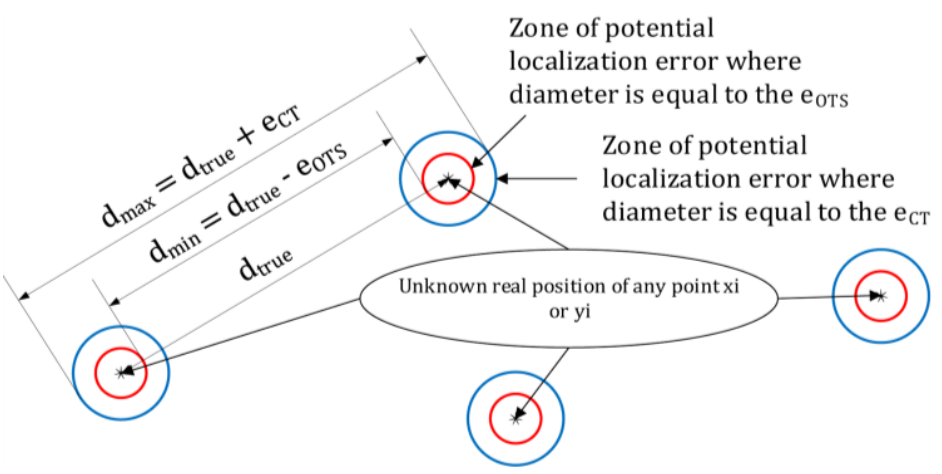

Figure 6: Potential zone of the fiducial localization error (FLE) with respect to the distance between any two fiducials.

The maximum distance error which is compared to $e$ in the correspondence algorithm is calculated as the maximum potential error caused by the FLE:

$$
e_{\text {max }}=d_{\text {max }}-d_{\text {min }}=d_{\text {true }}+e_{C T}-d_{\text {true }}-\left(-e_{\text {OTS }}\right)=e_{C T}+e_{\text {OTS }},
$$

where $e_{C T}$ can be viewed as the scalar value of $\mathbf{e}_{\mathrm{xi}}$ and $e_{O T S}$ as a scalar value of $\mathbf{e}_{\mathrm{yj}}$ in the situation of maximum error. To test the correspondence algorithm in a simulated environment, we need to calculate the distribution of $e_{O T S}$ and $e_{C T}$.

In our previous work [14], we developed the algorithm for the localization of fiducial markers in CT images. In the experiment of that study for calculation of $e_{C T}$, we used the Xshaped frame (shown in Fig. 1) with four fiducial markers which were localised in the CT scans of twelve patients and five CT scans of the test phantom. Six distances between fiducial markers of every CT scan were calculated and compared to those measured on the coordinate measuring machine (CMM). Separate statistics were calculated for the phantom and for the patient CT scans. The measured mean error for the patient CT scans was $0.4414 \mathrm{~mm}$, with a standard deviation of $0.56 \mathrm{~mm}$. The phantom CT scans demonstrated a significantly lower mean error value at $0.11 \mathrm{~mm}$ with a standard deviation of $0.10 \mathrm{~mm}$. The normality of distribution was confirmed by the Kolmogorov-Smirnov test.

The FLE in the OR is a result of the signal noise captured by the OTS and the resolution of cameras. In our previously published article [12], we measured the accuracy of the OTS Polaris Vicra (Nothern Digital), which was used for guiding the industrial robot arm to positions planned in the CT scan. The measured accuracy of the OTS was determined when the $\mathrm{x}$-shaped frame with four fiducial markers was used. For calculating $e_{\text {OTS }}$, we needed measurements for a single fiducial marker error. Wiles et al. [25] made an in-depth analysis of error distribution of the Polaris OTS. Over 1500 points were measured in the working area of the OTS by moving a single fiducial marker. The error between the ground truth data measured on the CMM and the positions of individual fiducial marker measured using the OTS showed an $R M S$ of $0.26 \mathrm{~mm}$, a mean error of $0.19 \mathrm{~mm}$, and a standard deviation of $0.17 \mathrm{~mm}$.

For the algorithm testing, we used coordinates of eight adhesive fiducial markers manually attached by the neurosurgeon to three patients and localized in the CT scans. To compensate for the difference in the anatomy of a human head and the positioning of fiducial markers in different surgeries, we used a uniform distribution on the coordinates of every fiducial marker inside a $10 \mathrm{~mm}$ radius sphere. The number of fiducial markers was $f \in[3,8]$. Randomly chosen fiducial markers were removed from the original set in every simulation when situations with less than eight markers were tested. The order of points in the second set was changed after the original set of points had been replaced. The noise was applied to both sets according to the calculated normal standard distribution, i.e. $0.56 \mathrm{~mm}$ on the original set and $0.17 \mathrm{~mm}$ on the second set. Furthermore, tests were run with $o \in[0,4]$ outlier points added in 
both sets. The positions of simulated outlier points were randomly chosen following a uniform distribution inside a sphere with a radius of $300 \mathrm{~mm}$ and with the centre defined as the centroid of the fiducial markers. Ten thousand simulations were performed for each of the three patients. For each combination of the number of fiducial markers $f \in[3,8]$, and each number of added outlier points $o \in[0,4]$, we ran ten thousand simulations, resulting in a total of 900000 tests. The algorithm was tested with the $e \in[1,6] \mathrm{mm}$ parameter and $0.1 \mathrm{~mm}$ step. There were three possible outcomes:

1. Successful correspondence - the exact number of points was corresponding and the returned order of points was identical to the known order of points in both sets,

2. Unsuccessful correspondence - due to big errors in the input data or an unambiguous solution,

3. False-positive result - when the algorithm returned the correspondence but the order of points was not correct when checked with the known order of points in both sets.

The results of correspondence algorithm testing for unsuccessful correspondence and a false-positive result are shown in Fig. 7.
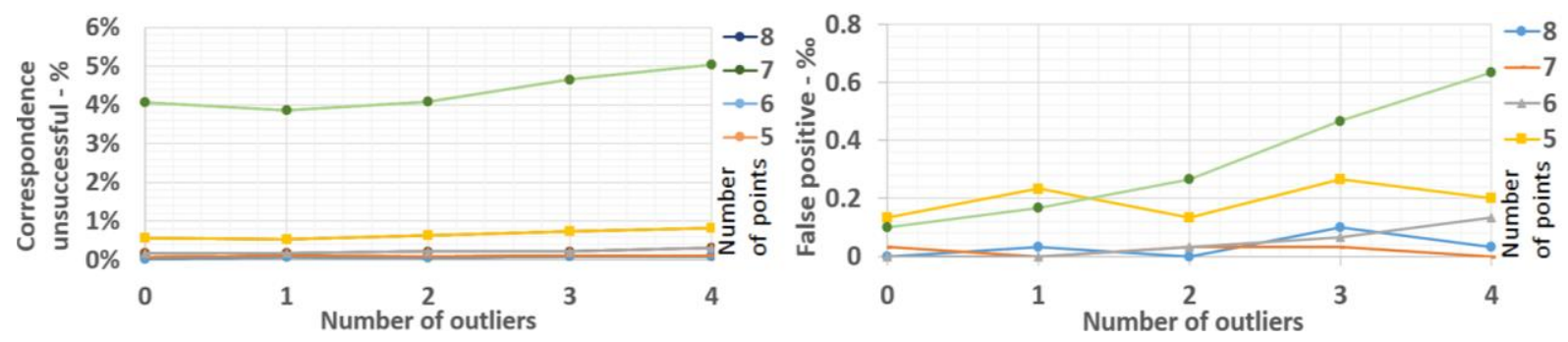

Figure 7: Results of the correspondence algorithm testing on simulated data; left: unsuccessful correspondence, right: false-positive result.

As expected, the correspondence algorithm has shown a higher percentage of success when a larger number of fiducial markers and a smaller number of outlier points were used. For five to eight fiducial markers and 0-4 outlier points, the percentage of unsuccessful correspondence was between $0-0.81 \%$ and the number of false-positive results was between $0-0.026 \%$. When only three or four fiducial markers were used, there was a higher chance of ambiguous solutions and hence a higher chance of unsuccessful correspondence and falsepositive results. The unsuccessful correspondence for four fiducial markers was 3.86-5.03\% and $7.98 \%-15.53 \%$ for three (not shown in Fig. 7.). False-positive results for four fiducial markers amounted to $0.01-0.06 \%$ and $0.05-0.89 \%$ for three. Based on the test results, in the case when three or four fiducial markers are used, we suggest that there should be a physical template which would enable unique positioning distances in the patient preparation procedure. This can be achieved with a three point physical template that can be attached to pretty much any shape and size of the human skull. The presented algorithm only needs three points to be placed in a unique spatial configuration to guarantee successful correspondence.

\subsection{Correspondence algorithm testing on clinical data}

The purpose of these tests was to verify the reliability of the developed correspondence algorithm with the parameter $e \in[1,6] \mathrm{mm}$ used on a real data set. The input data for the correspondence algorithm were the coordinates of the fiducial markers localized both in the physical and in the image space. In the image space, the fiducial markers were localized using the developed localization algorithm on CT scans of twelve patients done after a brain biopsy procedure and five CT scans of a laboratory phantom. In the physical space, the fiducial markers were localized using the Polaris Spectra OTS. We made 1415 measurements of the four fiducial markers mounted on the $\mathrm{x}$-shaped frame. During the data acquisition stage, the 
$\mathrm{x}$-shaped frame was constantly moved to ensure that different areas of the working volume are covered with the OTS. In Figs. 8 and 9, the percentage of successful correspondences is shown in relation to the parameter $e$ used in the algorithm.

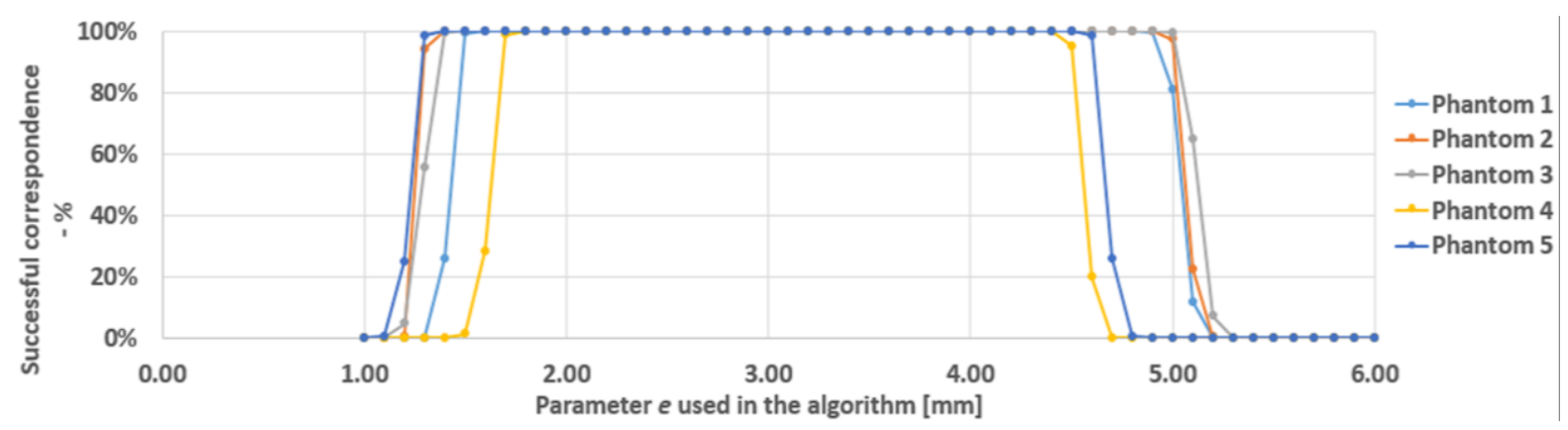

Figure 8: Success rate of the correspondence algorithm with the data from five CT scans of a laboratory phantom and OTS measurements.

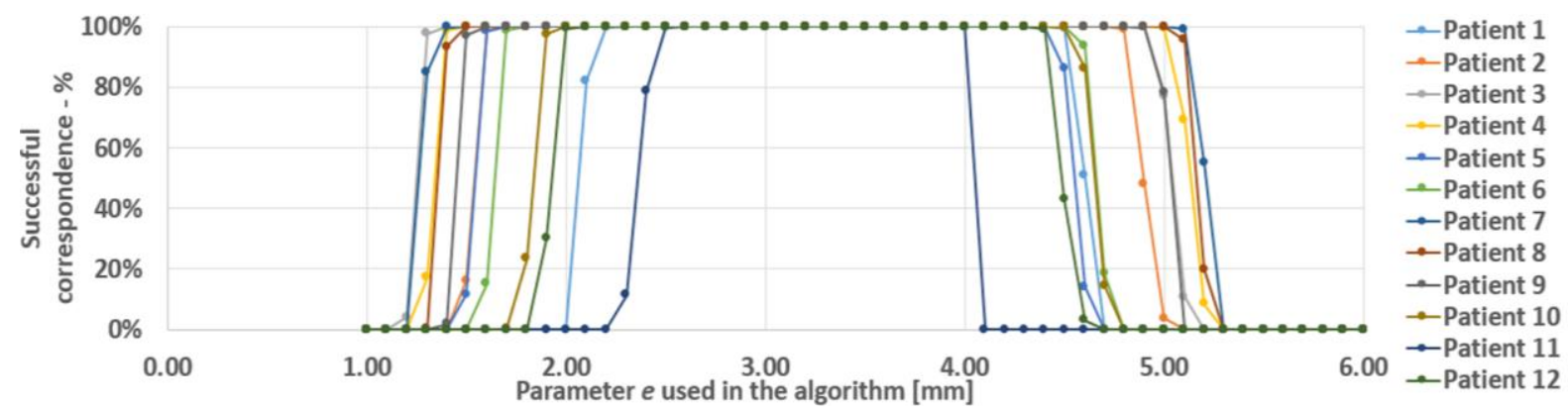

Figure 9: Success rate of the correspondence algorithm with the data from 12 patient CT scans and OTS measurements.

In 24055 tests, the success rate was $100 \%$. This can be contributed to the $\mathrm{x}$-shaped marker which ensured that four fiducial markers were positioned at unique distances from one to the other. In Figs. 8 and 9 one can note that the standard deviation of the errors in the phantom CT scans was lower than that in the patient CT scans and that the same OTS data was used in both cases. Consequently, there was a wider range of the applied parameter $e$ that yields successful correspondence results. All the phantom CT scans had a $100 \%$ success rate for the $e \in[1.8,4.5] \mathrm{mm}$ and all the patient CT scans for the $e \in[2.5,4] \mathrm{mm}$. There were no cases with unsuccessful correspondence or false-positive results in the specified interval of parameter $e$ because the highest value of $e$ was lower than those of the most similar distances between any two fiducial markers.

\section{CONCLUSION AND FUTURE WORK}

In this paper, we have introduced a framework for robotic neurosurgical patient registration using freely distributed fiducials with a high level of automation. Both the image space and the physical space localization, and subsequently the registration, are executed autonomously and do not require additional assistance of the medical personnel. We have also presented a novel correspondence algorithm that uses a similarity matrix to maximize the possibility of successful point pairing and to remove the potential outlier points. The test results have shown that the algorithm has a high level of reliability in determining the correct correspondence and in removing the outlier points. In the future, we aim to modify the algorithm so that it will be able to manage a larger number of fiducial points while maintaining the robustness to outlier points. With such improvements, the algorithm will become more suitable for a wider range of applications which use point cloud data. 
In the future research, we also plan to extend the use of robots into the preoperative patient preparation phase. At this point, the placement of fiducial markers onto the patient is performed by the neurosurgeon. Manual placement of fiducial markers can result in a bigger registration error due to the inadequate positioning of the markers with respect to the planned operative trajectories. West et al. [26] report that the registration error can be reduced if: more fiducial markers are used; fiducials are not placed in near-collinear configurations; and the centroid of all the fiducial points is closer to the target point. We plan to develop a robotic arm equipped with a 3D scanner, which would be able to perform marker-less registration of the patient's head and also with a tool for implanting or attaching fiducial markers. Once the patient is localized by the robot, the algorithm would calculate the best positions of the fiducial markers on the surface of the patient's head.

\section{ACKNOWLEDGEMENT}

The authors would like to acknowledge the support of the Croatian Scientific Foundation received through the research project ACRON (HRZZ-4192). The authors would also like to thank the entire team from the University Hospital Dubrava and especially Assistant Professor Darko Chudy and Domagoj Dlaka, dr. med. for the tremendous help in the clinical trials and procedures.

\section{REFERENCES}

[1] Moustris, G. P.; Hiridis, S. C.; Deliparaschos, K. M.; Konstantinidis, K. M. (2011). Evolution of autonomous and semi-autonomous robotic surgical systems: a review of the literature, The International Journal of Medical Robotics and Computer Assisted Surgery, Vol. 7, No. 4, 375392, doi: $10.1002 / \mathrm{rcs} .408$

[2] Mani, V. R. S.; Arivazhagan, S. (2013). Survey of medical image registration, Journal of Biomedical Engineering and Technology, Vol. 1, No. 2, 8-25

[3] Oliveira, F. P. M.; Tavares, J. M. R. S. (2014). Medical image registration: a review, Computer Methods in Biomechanics and Biomedical Engineering, Vol. 17, No. 2, 73-93, doi: $10.1080 / 10255842.2012 .670855$

[4] Widmann, G.; Schullian, P.; Ortler, M.; Bale, R. (2012). Frameless stereotactic targeting devices: technical features, targeting errors and clinical results, The International Journal of Medical Robotics and Computer Assisted Surgery, Vol. 8, No. 1, 1-16, doi:10.1002/rcs.441

[5] Cardinale, F.; Rizzi, M.; d’Orio, P.; Casaceli, G.; Arnulfo, G.; Narizzano, M.; Scorza, D.; De Momi, E.; Nichelatti, M.; Redaelli, D.; Sberna, M.; Moscato, A.; Castana, L. (2017). A new tool for touch-free patient registration for robot-assisted intracranial surgery: application accuracy from a phantom study and a retrospective surgical series, Neurosurgical Focus, Vol. 42, No. 5, Paper E8, 7 pages, doi:10.3171/2017.2.FOCUS16539

[6] De Benedictis, A.; Trezza, A.; Carai, A.; Genovese, E.; Procaccini, E.; Messina, R.; Randi, F.; Cossu, S.; Esposito, G.; Palma, P.; Amante, P.; Rizzi, M.; Marras, C. E. (2017). Robot-assisted procedures in pediatric neurosurgery, Neurosurgical Focus, Vol. 42, No. 5, Paper E7, 12 pages, doi:10.3171/2017.2.FOCUS16579

[7] González-Martínez, J.; Bulacio, J.; Thompson, S.; Gale, J.; Smithason, S.; Najm, I.; Bingaman, W. (2016). Technique, results, and complications related to robot-assisted stereoelectroencephalography, Neurosurgery, Vol. 78, No. 2, 169-180, doi:10.1227/ NEU.0000000000001034

[8] Lefranc, M.; Capel, C.; Pruvot, A. S.; Fichten, A.; Desenclos, C.; Toussaint, P.; Le Gars, D.; Peltier, J. (2014). The impact of the reference imaging modality, registration method and intraoperative flat-panel computed tomography on the accuracy of the ROSA ${ }^{\circledR}$ stereotactic robot, Stereotactic and Functional Neurosurgery, Vol. 92, No. 4, 242-250, doi:10.1159/000362936

[9] Kronreif, G.; Ptacek, W.; Kornfeld, M.; Fürst, M. (2012). Evaluation of robotic assistance in neurosurgical applications, Journal of Robotic Surgery, Vol. 6, No. 1, 33-39, doi:10.1007/ s11701-011-0327-y 
[10] Gerber, N.; Gavaghan, K. A.; Bell, B. J.; Williamson, T. M.; Weisstanner, C.; Caversaccio, M.D.; Weber, S. (2013). High-accuracy patient-to-image registration for the facilitation of imageguided robotic microsurgery on the head, IEEE Transactions on Biomedical Engineering, Vol. 60, No. 4, 960-968, doi:10.1109/TBME.2013.2241063

[11] Meng, F.; Ding, H.; Wang, G. (2014). A stereotaxic image-guided surgical robotic system for depth electrode insertion, $201436^{\text {th }}$ Annual International Conference of the IEEE Engineering in Medicine and Biology Society, 6167-6170, doi:10.1109/EMBC.2014.6945037

[12] Šuligoj, F.; Jerbić, B.; Švaco, M.; Šekoranja, B.; Mihalinec, D.; Vidaković, J. (2015). Medical applicability of a low-cost industrial robot arm guided with an optical tracking system, 2015 IEEE/RSJ International Conference on Intelligent Robots and Systems (IROS), 3785-3790, doi:10.1109/IROS.2015.7353908

[13] Lin, C.-C.; Lin, H.-C.; Lee, W.-Y.; Lee, S.-T.; Wu, C.-T. (2017). Neurosurgical robotic arm drilling navigation system, The International Journal of Medical Robotics and Computer Assisted Surgery, Vol. 13, No. 3, Paper e1790, doi:10.1002/rcs.1790

[14] Šuligoj, F.; Švaco, M.; Jerbić, B.; Šekoranja, B.; Vidaković, J. (2017). Automated marker localization in the planning phase of robotic neurosurgery, IEEE Access, Vol. 5, 12265-12274, doi:10.1109/ACCESS.2017.2718621

[15] Jerbic, B.; Nikolic, G.; Chudy, D.; Svaco, M.; Sekoranja, B. (2015). Robotic application in neurosurgery using intelligent visual and haptic interaction, International Journal of Simulation Modelling, Vol. 14, No. 1, 71-84, doi:10.2507/IJSIMM14(1)7.290

[16] Vidaković, J.; Jerbić, B.; Švaco, M.; Šuligoj, F.; Šekoranja, B. (2017). Position planning for collaborating robots and its application in neurosurgery, Technical Gazette, Vol. 24, No. 6, 1705 1711, doi:10.17559/TV-20170213110534

[17] Švaco, M.; Šekoranja, B.; Šuligoj, F.; Vidaković, J.; Jerbić, B.; Chudy, D. (2017). A novel robotic neuronavigation system: RONNA G3, Strojniski vestnik - Journal of Mechanical Engineering, Vol. 63, No. 12, 725-735, doi:10.5545/sv-jme.2017.4649

[18] Dlaka, D.; Švaco, M.; Chudy, D.; Jerbić, B.; Šekoranja, B.; Šuligoj, F.; Vidaković, J.; Almahariq, F.; Romić, D. (2018). Brain biopsy performed with the RONNA G3 system: a case study on using a novel robotic navigation device for stereotactic neurosurgery, The International Journal of Medical Robotics and Computer Assisted Surgery, Vol. 14, No. 1, Paper e1884, 7 pages, doi: $10.1002 / \mathrm{rcs} .1884$

[19] Yin, S.; Ren, Y.; Zhu, J.; Yang, S.; Ye, S. (2013). A vision-based self-calibration method for robotic visual inspection systems, Sensors, Vol. 13, No. 12, 16565-16582, doi:10.3390/ $\underline{\text { s131216565 }}$

[20] Bellekens, B.; Spruyt, V.; Berkvens, R.; Weyn, M. (2014). A survey of rigid 3D pointcloud registration algorithms, AMBIENT 2014: The Fourth International Conference on Ambient Computing, Applications, Services and Technologies, 8-13

[21] Besl, P. J.; McKay, N. D. (1992). A method for registration of 3-D shapes, IEEE Transactions on Pattern Analysis and Machine Intelligence, Vol. 14, No. 2, 239-256, doi:10.1109/34.121791

[22] Yaniv, Z. (2008). Rigid registration, Peters, T.; Cleary, K. (Eds.), Image-Guided Interventions, Springer, Boston, 159-192, doi:10.1007/978-0-387-73858-1 6

[23] Maurer Jr, C. R.; McCrory, J. J.; Fitzpatrick, J. M. (1993). Estimation of accuracy in localizing externally attached markers in multimodal volume head images, Medical Imaging 1993: Image Processing, Proc. SPIE, Vol. 1898, 43-54

[24] Fitzpatrick, J. M.; West, J. B.; Maurer Jr., C. R. (1998). Predicting error in rigid-body point-based registration, IEEE Transactions on Medical Imaging, Vol. 17, No. 5, 694-702, doi:10.1109/ $\underline{42.736021}$

[25] Wiles, A. D.; Thompson, D. G.; Frantz, D. D. (2004). Accuracy assessment and interpretation for optical tracking systems, Medical Imaging 2004: Visualization, Image-Guided Procedures, and Display, Proc. SPIE, Vol. 5367, 421-432, doi:10.1117/12.536128

[26] West, J. B.; Fitzpatrick, J. M.; Toms, S. A.; Maurer Jr, C. R.; Maciunas, R. J. (2001). Fiducial point placement and the accuracy of point-based, rigid body registration, Neurosurgery, Vol. 48, No. $4,810-816$ 